\title{
High energy conversion efficiency in laser-proton acceleration by controlling laser-energy deposition onto thin foil targets
}

C. M. Brenner, ${ }^{1,2}$ A. P. L. Robinson, ${ }^{2}$ K. Markey, ${ }^{2}$ R. H. H. Scott,${ }^{2}$ R. J. Gray, ${ }^{1}$ M. Rosinski, ${ }^{3}$ O. Deppert, ${ }^{4}$ J. Badziak, ${ }^{3}$ D. Batani, ${ }^{5}$ J. R. Davies, ${ }^{6}$ S. M. Hassan, ${ }^{7}$ K. L. Lancaster, ${ }^{2}$ K. $\mathrm{Li}^{8}{ }^{8}$ I. O. Musgrave, ${ }^{2}$ P. A. Norreys, ${ }^{2,9}$ J. Pasley, ${ }^{10,2}$ M. Roth, ${ }^{11}$ H-P. Schlenvoigt, ${ }^{12}$ C. Spindloe, ${ }^{2}$ M. Tatarakis, ${ }^{7}$ T. Winstone, ${ }^{2}$ J. Wolowski, ${ }^{3}$ D. Wyatt, ${ }^{2}$ P. McKenna, ${ }^{1}$ and D. Neely ${ }^{2}$

${ }^{1)}$ SUPA, Department of Physics, University of Strathclyde, Glasgow G4 ONG,

$U K$

${ }^{2)}$ Central Laser Facility, STFC, Rutherford Appleton Laboratory, Didcot, Oxon, OX11 OQX, $U K$

3) Institute of Plasma Physics and Laser Microfusion, 00-908 Warsaw, Poland

4) Institut für Kernphysik, Technische Universität Darmstadt, 64289 Darmstadt, Germany

5) Université Bordeaux, CNRS, CEA, CELIA (Centre Lasers Intenses et Applications), UMR 5107, Talence,

France

6) Fusion Science Center for Extreme States of Matter, Laboratory for Laser Energetics, University of Rochester, Rochester, New York 14623, USA

${ }^{7)}$ Centre for Plasma Physics and Lasers, Department of Electronics Engineering, 73133 Chania, 74100 Rethymno, Crete, Greece

8) GoLP, Instituto de Plasmas e Fusao Nuclear-Laboratrio Associado, Instituto Superior Tecnico, 1049-001 Lisboa, Portugal

${ }^{9)}$ Department of Physics, Blackett Laboratory, Imperial College, London, SW7 2BZ, $U . K$.

${ }^{10)}$ Department of Physics, University of York, York YO10 5DD, UK

${ }^{11)}$ Institut fur Kernphysik, Technische Universitat Darmstadt, 64289 Darmstadt, Germany

${ }^{12)}$ LULI, Ecole Polytechnique, 91128 Palaiseau, France

(Dated: 30 January 2014)

An all-optical approach to laser-proton acceleration enhancement is investigated using the simplest of target designs to demonstrate application-relevant levels of energy conversion efficiency between laser and protons. Controlled deposition of laser energy, in the form of a double-pulse temporal envelope, is investigated in combination with thin foil targets, in which recirculation of laser-accelerated electrons can lead to optimal conditions for coupling laser drive energy into the proton beam. This approach is shown to deliver a substantial enhancement in the coupling of laser energy to $5-30 \mathrm{MeV}$ protons, compared to single pulse irradiation, reaching a record high $15 \%$ conversion efficiency with a temporal separation of 1 ps between the two pulses and a $5 \mu \mathrm{m}$-thick Au foil. A 1D simulation code is used to support and explain the origin of the observation of an optimum pulse separation of $\sim 1 \mathrm{ps}$.

Proton acceleration driven by the interaction of an intense laser pulse and a solid target has received a considerable amount of attention over the last decade, see ref ${ }^{1}$ and references therein, and continues to be pursued because of the useful proton beam qualities, namely short pulse duration, low emmittance and high peak brightness, that can be obtained simultaneously. Furthermore, laser-driven proton beams produced in the interaction regime explored here demonstrate a broadband energy spectrum, which is suited to applications requiring volumetric dose deposition where a compact and flexible source is the ideal. In particular, isotope production ${ }^{2}$ and laser-driven fast-ignition fusion ${ }^{3}$ require proton beams of energy $<30 \mathrm{MeV}$ with ultra-high brightness and thus high conversion efficiency of laser energy into such a proton beam. For example, it has been noted that at least $15 \%$ energy conversion is required for proton fast ignition to be feasible ${ }^{3}$. High conversion efficiency ${ }^{4}$ has been reported but into proton beams with much higher maximum energy than is suitable for the appli- cations discussed here. Enhancement of proton beam brightness has been previously achieved with the use of structured $^{5}$, reduced thickness ${ }^{6,7}$ and reduced size ${ }^{8,9}$ targets as well as when controlled double-pulse interactions are employed ${ }^{10-12}$. The work reported here combines two conversion efficiency enhancing methods that are well suited for applications where a high pulse rate laser driver is envisioned: a) double-pulse interaction and b) a plain foil reduced thickness target, to achieve a laser-to-proton energy conversion efficiency that exceeds all other experimental measurements reported in the literature, to date.

Under the laser interaction conditions studied here, the target normal sheath acceleration mechanism (TNSA) ${ }^{13}$ is commonly used to describe the generation of a charge separation electric field, driven by laser-accelerated (fast) electrons, by which contaminant surface layers are ionised and protons are accelerated at the rear surface of the target ${ }^{14}$. Decreasing the target thickness to shorten the recirculation time of laser-accelerated electrons reflected by the sheath field at the target surfaces can extend 
the acceleration time ${ }^{9}$ while also increasing the area over which acceleration occurs, thus increasing the proton fluence. Numerical $^{15}$ and experimental ${ }^{12}$ work have also demonstrated that initiating pre-expansion of the rear surface by employing a controlled double-pulse configuration with picosecond temporal separation can be an effective technique for laser-to-proton energy conversion efficiency enhancement. A factor of three enhancement was observed when this method was employed with targets for which recirculation effects were negligible ${ }^{12}$.

Presented here are the first measurements of the laserto-proton conversion efficiency extracted when the double pulse mechanism is used in combination with recirculation in thin foil targets. Measurements were made during an experimental campaign performed using the petawatt arm of the Vulcan laser system. The 1054 nm Vulcan Petawatt laser was set-up to generate double pulses with an adjustable temporal separation of up to several picoseconds ${ }^{16}$. Laser pulses of FWHM duration $\sim$ 800 fs and intensity ratio $\mathrm{I}_{\text {initial }}: \mathrm{I}_{\text {main }}$ of $1: 10$ were delivered onto the target containing a total energy (regardless of the time delay) of $\sim 180 \mathrm{~J}$. The double pulse intensity ratio was chosen using the results of Markey et $a l^{12}$ and simulations which demonstrated that this ratio delivers a significant enhancement in the proton acceleration, compared to other combinations. The beam was focussed onto the target at $45^{\circ}$ incidence, in p-polarised geometry. The area of irradiation was set to $\sim 30 \mu \mathrm{m}$ so as to lower the effective laser intensity at the front surface of the target with the intention of decreasing the maximum energy of the accelerated proton beam to within the energy range of interest (5-25 MeV), whilst maintaining the maximum laser energy available for absorption into the fast electron population. Therefore, the intensity of the initial pulse was $\sim 3.2 \times 10^{18} \mathrm{~W} / \mathrm{cm}^{2}$, yielding a peak intensity of $\sim 2.9 \times 10^{19} \mathrm{~W} / \mathrm{cm}^{2}$ in the main drive pulse. Planar gold foil targets were irradiated using a temporally shaped laser intensity profile (double-pulse) with temporal separations $\left(t_{\text {delay }}\right)$ of 0 ps up to 4 ps between the pulses. Targets of thickness $5 \mu \mathrm{m}$ were used as this thickness is well within the recirculation regime $(<120$ $\mu \mathrm{m}$ for $800 \mathrm{fs}$ pulses) and represents a target choice that is simple to acquire and mount. The nanosecond and picosecond laser intensity contrast ratios were measured ${ }^{17}$ as $\sim 10^{10}$ and $\sim 10^{8}$, respectively.

The main diagnostic employed to record the forward accelerated proton beams was a $25 \mathrm{~mm} \times 50 \mathrm{~mm}$ stack of radiochromic dosimetry film $(\mathrm{RCF})$ placed $\sim 40 \pm 1 \mathrm{~mm}$ from the rear surface of the target. The RCF stack was positioned $\sim 1 \mathrm{~mm}$ below the laser axis so as to capture the lower half of the proton beam while allowing a line of sight for diagnostics employed to monitor other features of the laser interaction. Preparatory shots using pieces of $50 \mathrm{~mm} \times 50 \mathrm{~mm}$ RCF demonstrated that the laser accelerated proton beams were circular and symmetric about the laser axis and centered around the target normal direction ensuring that the half stack design was sufficient to provide information about the full beam. A $26 \mu \mathrm{m}-$ thick layer of Aluminium foil was included on the targetfacing side of the RCF stack to absorb accelerated ions, so that protons were the dominant species contributing to the dose measurement. For all shots the first layer of $\mathrm{RCF}$ was redundant due to saturation, which resulted in a minimum detectable proton energy of $3.3 \mathrm{MeV}$ for the diagnostic. The RCF signal was converted to proton dose signal ${ }^{18}$ using a calibration against films irradiated with known dose up to $200 \mathrm{kGy}$ using the University of Birmingham cyclotron. The proton dose-energy spectrum given by the RCF diagnostic was deconvolved ${ }^{19}$ to take into account the contribution to the measured dose of all proton energies that had passed through the RCF pieces, to give the number of protons per $\mathrm{MeV}$ as a function of proton energy.

Comparing the proton spectra obtained using a double-pulse interaction to the single pulse (0 ps $\left.t_{\text {delay }}\right)$ proton spectrum in figure 1 reveals that the higher energy portions $(>15 \mathrm{MeV})$ of the beams exhibit a similar temperature, indicated by the slope of the spectrum. However, when the double-pulse mechanism is employed using a pulse separation of 1 ps a significant enhancement in the proton number over the whole spectrum is achieved. There is a factor of $4-8$ increase in the fluence of protons with energy $\sim 3-15 \mathrm{MeV}$, with the highest increase for the lowest proton energies recorded. The enhancement in the proton spectrum in this energy range when the double pulse is employed with $t_{\text {delay }}=$ $2 \mathrm{ps}$ is slight, compared to using $t_{\text {delay }}=1 \mathrm{ps}$, and there appears to be no enhancement when the pulse delay is increased further, but instead a noticeable decrease in proton number for energies $>15 \mathrm{MeV}$.

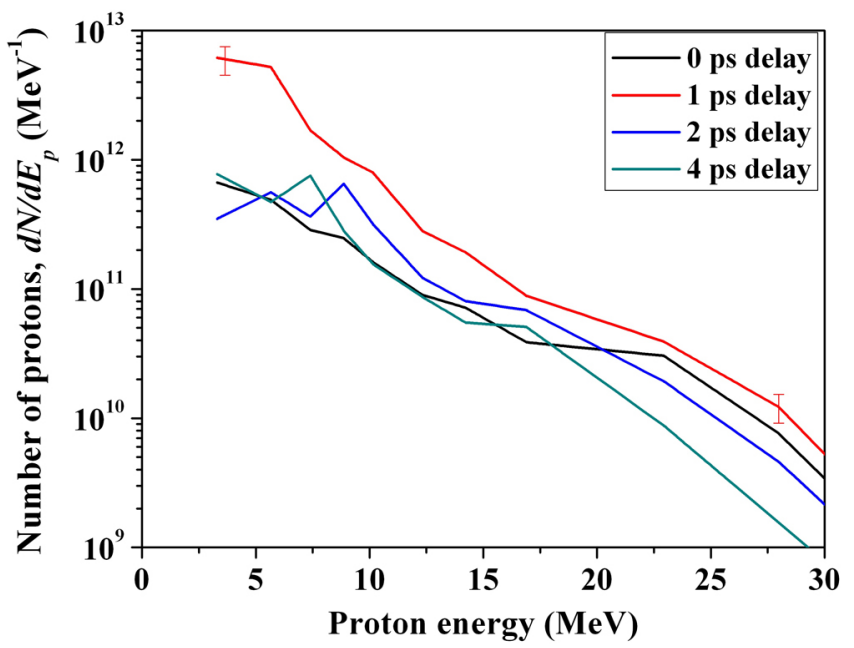

FIG. 1. Proton energy spectra obtained from $5 \mu$ m-thick targets with single pulse $\left(t_{\text {delay }}=0 \mathrm{ps}\right)$ and double-pulse $\left(t_{\text {delay }}=1,2\right.$ and $\left.4 \mathrm{ps}\right)$ irradiation. Sample error bars are shown for the 1 ps delay spectrum.

The proton spectra were used to calculate the total beam energy content within the detector energy range. Image analysis software was used to monitor the outer 
and inner contours of the beam at various layers in the stack in order to calculate the percentage of the beam captured by the diagnostic for each shot. A variation between shots of $6 \%$ in the fraction of the beam captured by the diagnostic was found, likely due to slight beam pointing fluctuations. The proton beam energy content was then compared to the laser energy incident on to the target for every shot, to give the conversion efficiency of laser energy into proton energy during the acceleration. Figure 2 demonstrates that a conversion efficiency of $15.1 \pm 2.6 \%$ to protons with energy $3.3 \mathrm{MeV}<E_{p}<$ $30.0 \mathrm{MeV}$ is achieved by combining a $5 \mu \mathrm{m}$ thick foil with a 1 ps separation double-pulse laser drive, indicating an energy content of $\sim 25 \mathrm{~J}$ within the proton beam. For reference, the laser-to-proton energy conversion efficiencies measured during similar conditions but with thicker foils $(100 \mu \mathrm{m})$ by Markey et al has also been included in figure 2, demonstrating the significant increase in conversion efficiency obtained by decreasing the thickness of the foil target to within the recirculating regime, as expected from previous work ${ }^{6}$.

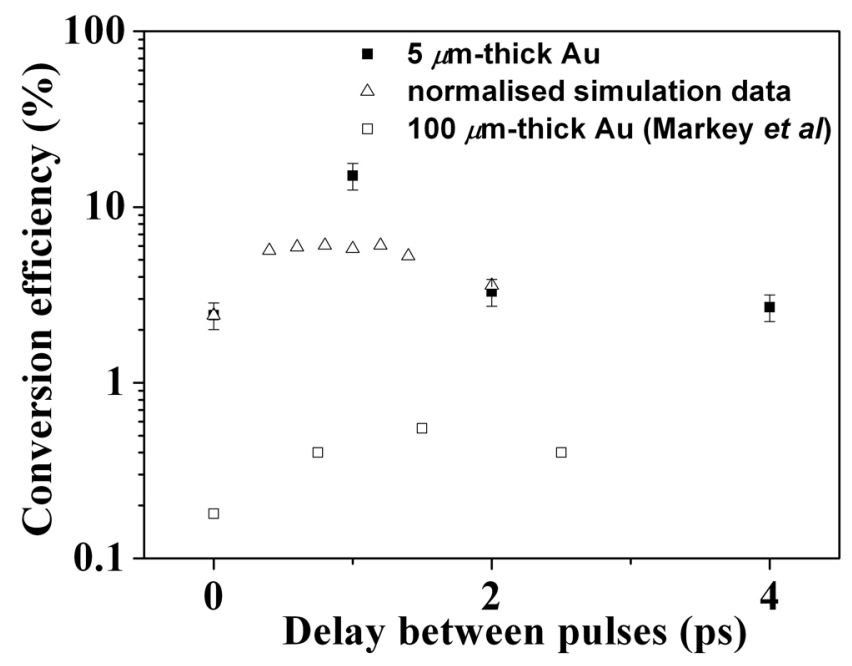

FIG. 2. Laser-to-proton energy conversion efficiency with respect to $t_{\text {delay }}$ obtained for $5 \mu \mathrm{m}$-thick Au targets compared to results obtained with thicker $(100 \mu$ m-thick $)$ Au targets. Simulated laser-to-proton energy conversion efficiency with respect to $t_{\text {delay }}$ normalised to the single pulse measured value is also shown.

Figure 2 clearly demonstrates that the double-pulse acceleration mechanism is optimised with a temporal separation of $\sim 1$ ps for the conditions studied here and that the effect falls off sharply with increasing $t_{\text {delay }}$. To investigate the underlining mechanisms determining the optimum pulse separation, particle-in-cell simulations using an explicit 1D3P code $^{15}$ have been carried out to explore the double-pulse laser drive effect on the coupling of energy between the fast electrons and lower-energy $(<10$ $\mathrm{MeV}$ ) forward-accelerated protons. A series of simulations were carried out within a grid of size $1200 \mu \mathrm{m}$ and cell width $3 \times 10^{-9} \mathrm{~m}$ using $2 \times 10^{6}$ macroparticles for each species. The target was a $5 \mu \mathrm{m}$-thick foil with an electron density of $2 \times 10^{28} \mathrm{~m}^{-3}$, made up of ions of charge $\mathrm{Z}=1$ and charge-to-mass ratio 0.01 so as to simulate a low charge-state heavy ion (gold-like) background while reducing the number of electrons needed, with a $20 \mathrm{~nm}$ thick layer of protons on each surface. To accommodate computational restraints, the laser duration was reduced which, despite leading to lower maximum proton energies than in the experiment, was long enough to establish a proton spectrum in the $<10 \mathrm{MeV}$ energy region of interest and enabled the effect on the proton acceleration to be studied as a function of temporal separation of fast electron injection. Two laser pulses, each of 200 fs FWHM duration, were initiated with various temporal separation between the peak of the pulses up to 2 ps. The intensity ratio and main-pulse peak intensities in the double and single pulse interactions were the same as the experimental values. The energy density within the simulated single and double pulse profiles was measured as being equal and the target was positioned at the foot of the first interacting pulse so as to replicate the steep plasma gradient interactions of the experiment. The energy content of the lower energy portion of the forwardaccelerated proton beam was extracted 400 fs after the peak of the main drive pulse had reached the target.

Plotting the energy conversion efficiency of the simulated single and double-pulse accelerated proton beams normalised to the measured single pulse value as a function of $t_{\text {delay }}$ (see figure 2) shows that the energy conversion is optimised for $t_{\text {delay }}$ between $800-1200 \mathrm{fs}$. This is in good agreement with the $1 \mathrm{ps}$ optimal pulse separation observed in the measured values, also shown in figure 2 for comparison. Despite there being agreement in the temporal evolution of the efficiency enhancement, the simulated efficiency enhancement is notably lower than observed in the experiment. This is likely due to the benefits of recirculation not being fully exploited in the simulations due to fewer fast-electron recirculations taking place within the shorter pulse duration employed, as well as front surface laser absorption mechanisms not being fully simulated in the $1 \mathrm{D}$ code. The efficiency enhancement decreases for both the measured and simulated values when $t_{\text {delay }}$ is increased to 2 ps. However, the simulations suggest that the range of temporal separations for which a considerable gain in conversion efficiency might be achieved is wide, between 400-1400 fs.

In order to establish the mechanism governing the optimisation with respect to the temporal separation, the simulated proton phase-space plots, particle densities and longitudinal electric fields for each simulation were analysed at $100 \mathrm{fs}$ time intervals after the peak of the main pulse had reached the front side of the target, at time $t_{0}$. For the double pulse interactions, a significant gain in the momentum of the lowest energy protons in close proximity to the heavy ion front is observed at times subsequent to $t_{0}$, see figure $3 \mathrm{a}$. The region over which this momentum gain occurs coincides with the region in which the proton density is highest (see figure $3 \mathrm{~b}$ ), indicating 


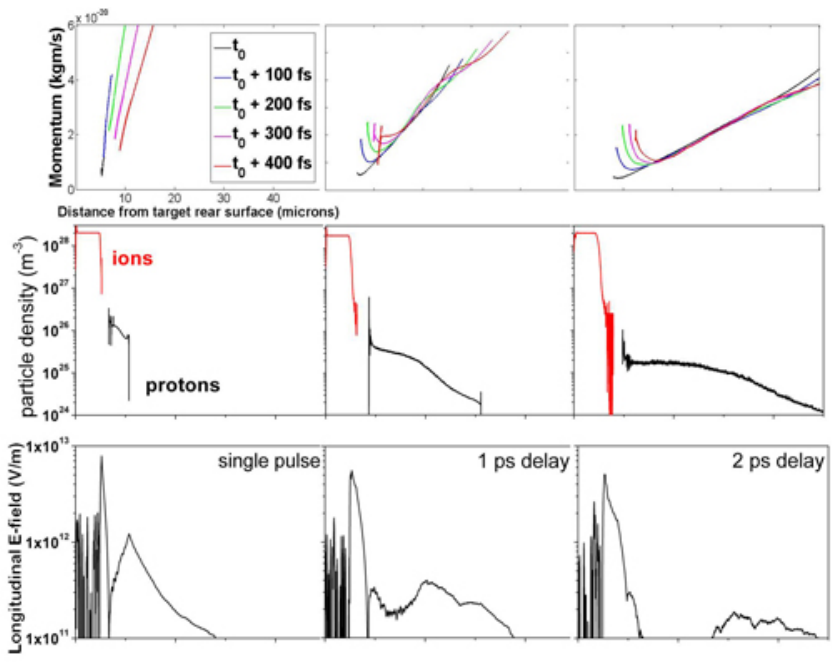

FIG. 3. a) Proton phase space plots (top) for $t_{\text {delay }}=0 \mathrm{ps}$ (left), 1ps (middle) and $2 \mathrm{ps}$ (right) extracted at $t_{0}$ and at 100 fs subsequent intervals, b) heavy ion and proton density plots (middle) and c) longitudinal electric field plots (bottom) extracted $200 \mathrm{fs}$ after the peak of the second pulse had reached the target front surface are also shown.

not only a boost in velocity of this proton population but also that the total energy content of the population was significantly boosted. This high density region is established as a result of a piston-like action due to electrostatic shock ${ }^{20}$ induced by the presence of the dense, heavy ion front expanding behind it. The proton density spike in the 1 ps simulation (middle column of figure 3 ) at the back of the proton population is the crucial difference between the three scenarios. The resultant $\sim$ order of magnitude density enhancement increase over the single pulse and $t_{\text {delay }}=2$ ps double pulse interactions, as well as a sharp drop in the density at the centre of the population, results in significant E-field effective across the whole proton population, with peaks in these high gradient regions (see figures $3 \mathrm{~b}$ and $3 \mathrm{c}$ ). The additional acceleration mechanism of ion-front repulsion in combination with fast-electron sheath acceleration is clearly significantly effective when this proton density profile is established, optimising the conversion of energy to protons and translating to an enhancement in the fluence of moderate energy protons observed.

The proton density plot for the single pulse interaction indicates that the peak in the E-field associated with the sheath field occurs at the leading edge of the expanding population where the proton density is lowest, which is a common feature of the single pulse sheath acceleration mechanism $^{21}$. In the case of a double pulse interaction with a longer delay of $2 \mathrm{ps}$, the proton population has sufficiently expanded so as to dampen the density gradients at both the front and centre of the proton population, thus the E-field enhancement in these regions will have neglible effect. The competing effects of time taken for the density spike to form as the heavy ion front expands and the expanding proton front dampening out the density gradients results in there being an optimum pulse delay for laser-to-proton conversion efficiency. For the conditions studied here, both the measured and simulated values concur that a delay of $\sim 0.8-1.2 \mathrm{ps}$ is optimum.

In conclusion, this paper demonstrates the benefit of employing the double-pulse sheath acceleration mechanism in combination with thin foils and that $15 \%$ conversion efficiency between laser and proton energy can be achieved when the pulse separation is optimised. This record high result shows that laser-driven proton sources are viable for use in heating and transmutation applications for which previously observed $<10 \%$ conversion efficiencies had been a severe constraint. Furthermore, simulations of the double-pulse laser interaction with a thin, solid foil made up of heavy ions suggest that an enhancement will be observed over a wide range of pulse separations from 0.4-1.4 ps. However when the pulse separation is $\geq 2 \mathrm{ps}$, the proton density gradients in the expanding front become shallower and the benefit of using the double-mechanism begins to decrease. The doublepulse mechanism of laser-proton acceleration is a simple but effective technique for controlling the properties of a sheath accelerated proton beam and is very promising for the envisioned applications. The enhancement could be improved further still by optimising laser parameters such as pulse duration and/or focal spot size as well as investigating with target design and composition. However, maintaining an all optical approach to the enhancement mechanism would be better suited for high repetition rate laser systems.

The authors gratefully acknowledge the expert staff and support of the Central Laser Facility. This work was undertaken as part of the HiPER preparatory project and supported by the UK's Science and Technology Facilities Council. This work is financially supported by EPSRC (grant numbers EP/J003832/1 and $\mathrm{EP} / \mathrm{K} 022415 / 1)$. The research leading to these results has also received funding from LASERLAB-EUROPE (grant agreement number 284464, EC's Seventh Framework Programme).

${ }^{1}$ H. Daido, M. Nishiuchi and A. S. Pirozchov, Rep. Prog. Phys. 75, 056401 (2012).

${ }^{2}$ K. Ledingham, P. McKenna, T. McCanny, S. Shimizu, J. Yang, L. Robson, J. Zweit, M. J. Gillies, J. Bailey, G. Chimon, et al., J. Phys. D Appl. Phys. 37, 2341, (2004).

${ }^{3}$ M. Roth, T. Cowan, M. Key, S. Hatchett, C. Brown, W. Fountain, J. Johnson, D. Pennington, R. Snavely, S. Wilks, et al., Phys. Rev. Lett. 86 436, (2001).

${ }^{4}$ R. A. Snavely, M. H. Key, S. P. Hatchett, T. E. Cowan, M. Roth, T. W. Phillips, M . A. Stoyer, E. A. Henry, T. C. Sangster, M. S. Singh, et al., Phys. Rev. Lett. 85, 2945, (2000).

${ }^{5}$ K. A. Flippo, E. D'Humières, S. A. Gaillard, J. Rassuchine, D. C. Gautier, M. Schollmeier, F. Nürnberg, J. L. Kline, J. Adams, B. Albright, et al., Phys. Plasmas 15, 056709, (2008).

${ }^{6}$ J. Fuchs, P. Antici, E. D'Humières, E. Lefebvre, M. Borghesi, E. Brambrink, C. A. Cecchetti, M. Kaluza, V. Malka, M. Manclossi, et al., Nature Phys. 2, 48, (2005).

${ }^{7}$ D. Neely, P. Foster, A. P. L. Robinson, F. Lindau, O. Lundh, A. 
Persson, C. -G. Wahlström and P. McKenna, App. Phys. Lett. 89, 021502 (2006).

${ }^{8}$ O. Tresca, D. C. Carroll, X. H. Yuan, B. Aurand, V. Bagnoud, C. M. Brenner, M. Coury, J. Fils, R. J. Gray, T. Kühl, et al., Plasma Phys. Contr. F. 53, 105008, (2011).

${ }^{9}$ S. Buffechoux, J. Psikal, M. Nakatsutsumi, L. Romagnani, A. Andreev, K. Zeil, M. Amin, P. Antici, T. Burris-Mog, A. CompantLa-Fontaine, et al., Phys. Rev. Lett. 105, 015005, (2010).

${ }^{10}$ P. McKenna, D. C. Carroll, O. Lundh, F. Nürnberg, K. Markey, S. Bandyopadhyay, D. Batani, R. G. Evans, R. Jafer, S. Kar, et al., Laser Part. Beams. 26, 591 (2008).

${ }^{11}$ F. Dollar, T. Matsuoka, G. M. Petrov, A. G. R. Thomas, S. S. Bulanov, V. Chvykov, J. Davis, G. Kalinchenko, C. McGuffey, L. Willingdale, et al., Phys. Rev. Lett. 107, 065003, (2011).

${ }^{12}$ K. Markey, P. McKenna, C. M. Brenner, D. C. Carroll, M. M. Günther, K. Harres, S. Kar, K. Lancaster, F. Nürnberg, M. N. Quinn, et al., Phys. Rev. Lett. 105, 195008 (2010).

${ }^{13}$ S. C. Wilks, A. B. Langdon, T. E. Cowan, M. Roth, M. Singh,
S. Hatchett, M. H. Key, D. Pennington, A. MacKinnon, and R. A. Snavely, Phys. Plasmas 8, 542 (2001).

${ }^{14}$ E. L. Clark, K. Krushelnick, M. Zepf, F. N. Beg, M. Tatarakis, A. Machacek, M. I. K. Santala, I. Watts, P. A. Norreys and A. E. Dangor, Phys. Rev. Lett. 85, 1654 (2000).

${ }^{15}$ A. P. L. Robinson, D. Neely, P. McKenna, and R. G. Evans, Plasma Phys. Contr. F. 49, 373 (2007).

${ }^{16}$ I. O. Musgrave, Central Laser Facility Annual Report, 1, (2011).

${ }^{17}$ W. Shaikh, I. O. Musgrave, M. Galimberti and A. Boyle, SPIE Proc. Ser., 8240, 82400T, (2012).

${ }^{18}$ P. R. Bolton, M. Borghesi, C. M. Brenner, D. C. Carroll, C. DeMartinis, A. Flacco, V. Floquet, J. Fuchs, P. Gallegos, D. Giove, et al., Phys. Medica, (2013).

${ }^{19}$ E. Breschi, M. Borghesi, M. Galimberti, D. Giulietti, L. A. Gizzi and L. Romagnani, Nucl. Instr. and Meth. A, 522, 190, (2004).

${ }^{20}$ L. O. Silva, M. Marti, J. R. Davies, R. A. Fonseca, C. Ren, F. S. Tsung and W. B. Mori, Phys. Rev. Lett. 92, 015002 (2004).

${ }^{21}$ P. Mora, Phys. Rev. Lett. 90, 185002 (2003). 